\title{
Existence of Positive Solutions for a Class of Nonlinear Algebraic Systems
}

\author{
Yongqiang Du, ${ }^{1}$ Guang Zhang, ${ }^{1}$ and Wenying Feng ${ }^{2}$ \\ ${ }^{1}$ School of Science, Tianjin University of Commerce, Tianjin 300134, China \\ ${ }^{2}$ Department of Mathematics, Department of Computing and Information Systems, Trent University, \\ Peterborough, ON, Canada K9J 7B8
}

Correspondence should be addressed to Guang Zhang; qd_gzhang@126.com

Received 28 October 2015; Revised 29 December 2015; Accepted 30 December 2015

Academic Editor: Evangelos J. Sapountzakis

Copyright (C) 2016 Yongqiang Du et al. This is an open access article distributed under the Creative Commons Attribution License, which permits unrestricted use, distribution, and reproduction in any medium, provided the original work is properly cited.

Based on Guo-Krasnoselskii's fixed point theorem, the existence of positive solutions for a class of nonlinear algebraic systems of the form $x=G F(x)$ is studied firstly, where $G$ is a positive $n \times n$ square matrix, $x=\operatorname{col}\left(x_{1}, x_{2}, \ldots, x_{n}\right)$, and $F(x)=$ $\operatorname{col}\left(f\left(x_{1}\right), f\left(x_{2}\right), \ldots, f\left(x_{n}\right)\right)$, where, $F(x)$ is not required to be satisfied sublinear or superlinear at zero point and infinite point. In addition, a new cone is constructed in $R^{n}$. Secondly, the obtained results can be extended to some more general nonlinear algebraic systems, where the coefficient matrix $G$ and the nonlinear term are depended on the variable $x$. Corresponding examples are given to illustrate these results.

\section{Introduction}

Many problems arising from economics [1-4], complex network [5-7], mechanical engineering [8], Verschelde's web page [9], and mathematics [10-13] can be formulated as a system of equations. The existence problem of a solution for a system of equations is called the zero point problem. This problem is associated with a function $f: X \rightarrow R^{n}$, where $X$ is a subset of the $n$-dimensional Euclidean space $R^{n}$. A point $x \in X$ is a zero point of $f$ if the image of $x$ is the origin, and $x$ is a fixed point of $f$ if $x$ is a zero point of the function $g$ given by $g(x)=f(x)-x$ and thus the image $f(x)$ of $x$ is $x$ itself.

Generally, there are no good methods for solving such systems, even in the simple case of only two equations of the form: $f_{1}\left(x_{1}, x_{2}\right)=0$ and $f_{2}\left(x_{1}, x_{2}\right)=0$; see van der Laan et al. [14]. Thus, some existing theorems of zero points or fixed points had been extensively established by a number of authors; see $[1,2,4,10-13,15-23]$ and so forth.

In applications, positive solutions for a system of equations are important; see [1, 2, 4-7, 9-13, 17-23, 23-29]. For example, in the study of discrete dynamical systems [5] or continuous time dynamical systems [6, 7], a positive solution for a system of equations represents the existence of stationary states. In another example, the second-order boundary value problem that represents an adiabatic tubular chemical reactor [27] can be changed to the form of a system of equations by using the SINC method for numerical solutions [29]. Positive solutions for a system of equations then represent the steady state temperature of the reaction. A positive solution for the system of equations also ensures the solvability of the three-point boundary value problem studied in [13].

Recently, in [12] the authors had considered the existence of positive solutions for the discrete Dirichlet boundary value problem of the form

$$
\begin{aligned}
\Delta^{2} x_{i-1}+f\left(x_{i}\right) & =0, \quad i \in[1, n], \\
x_{0} & =0=x_{n+1}
\end{aligned}
$$

by using Guo-Krasnoselskii's fixed point theorem, where $n$ is a positive integer, $[1, n]=\{1,2, \ldots, n\}$, and $\Delta$ is the forward difference operator; that is, $\Delta x_{i-1}=x_{i}-x_{i-1}$, and $\Delta^{2} x_{i-1}=$ $\Delta\left(\Delta x_{i-1}\right)$. 
Let $x=\operatorname{col}\left(x_{1}, x_{2}, \ldots, x_{n}\right), F(x)=\operatorname{col}\left(f\left(x_{1}\right), f\left(x_{2}\right), \ldots\right.$, $\left.f\left(x_{n}\right)\right)$, and $G=\left(g_{i j}\right)$, which is given by

$$
g_{i j}= \begin{cases}\frac{(n-i+1) j}{n+1}, & 1 \leq j \leq i \leq n, \\ \frac{(n-j+1) i}{n+1}, & 1 \leq i \leq j \leq n\end{cases}
$$

Then problem (1) can be rewritten by matrix and vector of the form

$$
x=G F(x)
$$

or

$$
x_{i}=\sum_{j=1}^{n} g_{i j} f\left(x_{j}\right) \quad \text { for } i \in[1, n] \text {. }
$$

Note that $m=\min _{i, j \in[1, n]}\left\{g_{i j}\right\}>0$ (where $g_{i j}$ is defined by (2)); thus, we can let $P=\left\{x: x_{i} \geq \delta|x|, x \in R^{n}\right\}$; then $P \subset R^{n}$ is a cone, where

$$
\begin{aligned}
|x| & =\max _{i \in[1, n]}\left\{\left|x_{i}\right|\right\}, \\
\delta & =\frac{m}{M}, \\
m & =\min _{i, j \in[1, n]}\left\{g_{i j}\right\}, \\
M & =\max _{i, j \in[1, n]}\left\{g_{i j}\right\} .
\end{aligned}
$$

In this case, the method in [12] is valid for a more general nonlinear algebraic system (3) when the coefficient matrix $G$ is positive; that is, all elements of $G$ are positive.

The more general nonlinear algebraic system (3) has been shown to have interesting applications in various areas such as difference equations, boundary value problems, dynamical networks, existence of periodic solutions, stochastic processes, and numerical analysis; see [5-7, 10-13, 27, 29] and the references therein.

In this paper, we will construct a new cone in $R^{n}$ and obtain some new existence results for a general nonlinear algebraic system (3). In particular, the obtained results are also sharp even if $n=1$. Naturally, we also improve our recent result when system (3) is reduced to problem (1). We will also extend our results to the more general cases (the coefficient matrix is dependent on the state variable $x$ or $\left.F(x)=\operatorname{col}\left(f_{1}(x), f_{2}(x), \ldots, f_{n}(x)\right)\right)$. In this case, all obtained results are new.

Our main tool is Guo-Krasnoselskii's fixed point theorem. Thus, in the next section, we give the theorem and prove three extended results. Our main results will be discussed in Section 3 and some more general cases will be considered in Section 4. Of course, some explanatory examples and remarks are also given in Sections 3 and 4, respectively.

\section{Preliminaries}

Guo-Krasnoselskii's fixed point theorem is mainly adopted in this paper. To this end, we will display this result in the following lemma. Firstly, we give a definition of cone. We let $E$ be a real Banach space. A nonempty closed convex set $P \subset E$ is called a cone if it satisfies the following two conditions: (i) $x \in P$ and $\lambda \geq 0$ imply that $\lambda x \in P$, and (ii) $x \in P$ and $-x \in P$ imply that $x=\theta$, where $\theta \in E$ is called the zero element of $E$.

Now we state Guo-Krasnoselskii's fixed point theorem concerning cone expansion and compression of norm type as follows.

Lemma 1 (see [30, P133]). Let $\Omega_{1}$ and $\Omega_{2}$ be two bounded open sets in $E$ such that $\theta \in \Omega_{1}$ and $\bar{\Omega}_{1} \subset \Omega_{2}$. Suppose that $A: P \cap$ $\left(\bar{\Omega}_{2} \backslash \Omega_{1}\right) \rightarrow P$ is completely continuous. If either $\left(H_{1}\right)\|A x\| \leq$ $\|x\|$ for $x \in P \cap \partial \Omega_{1}$ and $\|A x\| \geq\|x\|$ for $x \in P \cap \partial \Omega_{2}$ or $\left(H_{2}\right)\|A x\| \geq\|x\|$ for $x \in P \cap \partial \Omega_{1}$ and $\|A x\| \leq\|x\|$ for $x \in$ $P \cap \partial \Omega_{2}$ hold, then $A$ has at least one fixed point in $P \cap\left(\bar{\Omega}_{2} \backslash \Omega_{1}\right)$.

By using Lemma 1, we immediately obtain the following results.

Corollary 2. Let $\Omega_{1}$ and $\Omega_{2}$ be two bounded open sets in $E$ such that $\theta \in \Omega_{1}$ and $\bar{\Omega}_{1} \subset \Omega_{2}$. Suppose that $A: P \cap\left(\bar{\Omega}_{2} \backslash\right.$ $\left.\Omega_{1}\right) \rightarrow P$ is completely continuous. If either $\left(H_{1}^{1}\right)\|A x\|<\|x\|$ for $x \in P \cap \partial \Omega_{1}$ and $\|A x\| \geq\|x\|$ for $x \in P \cap \partial \Omega_{2}$ or $\left(H_{2}^{1}\right)\|A x\|>\|x\|$ for $x \in P \cap \partial \Omega_{1}$ and $\|A x\| \leq\|x\|$ for $x \in P \cap \partial \Omega_{2}$ hold, then $A$ has at least one fixed point in $P \cap\left(\bar{\Omega}_{2} \backslash \bar{\Omega}_{1}\right)$.

Corollary 3. Let $\Omega_{1}$ and $\Omega_{2}$ be two bounded open sets in $E$ such that $\theta \in \Omega_{1}$ and $\bar{\Omega}_{1} \subset \Omega_{2}$. Suppose that $A: P \cap\left(\bar{\Omega}_{2} \mid\right.$ $\left.\Omega_{1}\right) \rightarrow P$ is completely continuous. If either $\left(H_{1}^{2}\right)\|A x\| \leq\|x\|$ for $x \in P \cap \partial \Omega_{1}$ and $\|A x\|>\|x\|$ for $x \in P \cap \partial \Omega_{2}$ or $\left(H_{2}^{2}\right)\|A x\| \geq\|x\|$ for $x \in P \cap \partial \Omega_{1}$ and $\|A x\|<\|x\|$ for $x \in P \cap \partial \Omega_{2}$ hold, then $A$ has at least one fixed point in $P \cap\left(\Omega_{2} \backslash \Omega_{1}\right)$.

Corollary 4. Let $\Omega_{1}$ and $\Omega_{2}$ be two bounded open sets in $E$ such that $\theta \in \Omega_{1}$ and $\bar{\Omega}_{1} \subset \Omega_{2}$. Suppose that $A: P \cap\left(\bar{\Omega}_{2} \backslash\right.$ $\left.\Omega_{1}\right) \rightarrow P$ is completely continuous. If either $\left(H_{1}^{3}\right)\|A x\|<\|x\|$ for $x \in P \cap \partial \Omega_{1}$ and $\|A x\|>\|x\|$ for $x \in P \cap \partial \Omega_{2}$ or $\left(H_{2}^{3}\right)\|A x\|>\|x\|$ for $x \in P \cap \partial \Omega_{1}$ and $\|A x\|<\|x\|$ for $x \in P \cap \partial \Omega_{2}$ hold, then $A$ has at least one fixed point in $P \cap\left(\Omega_{2} \backslash \bar{\Omega}_{1}\right)$.

Indeed, by means of Lemma 1 , there exists $x^{*} \in P \cap\left(\bar{\Omega}_{2}\right)$ $\left.\Omega_{1}\right)$ such that $x^{*}=A x^{*}$. When all conditions of Corollary 2 hold, we have $\|A x\|<\|x\|$ or $\|A x\|>\|x\|$ for $x \in P \cap \partial \Omega_{1}$, which implies that $x^{*} \notin \partial \Omega_{1}$. Thus, $x^{*} \in P \cap\left(\bar{\Omega}_{2} \backslash \bar{\Omega}_{1}\right)$. If all conditions of Corollaries 3 and 4 hold, the proofs are similar.

\section{Existence of Positive Solutions for System (3)}

First of all, we give two conditions of Theorem 5 .

$\left(\mathrm{A}_{1}\right)$ There exists $\delta \in(0,1]$ and a positive sequence $\left\{\varphi_{j}\right\}_{j=1}^{n}$, such that $\delta \varphi_{j} \leq g_{i j} \leq \varphi_{j}$ for $i, j \in[1, n]$. 
$\left(\mathrm{A}_{2}\right)$ There exist $0<a<b$, such that the function $f$ : $[\delta a, b] \rightarrow R_{+}$is continuous and either

$$
\begin{array}{r}
\max _{s \in[\delta a, a]} f(s) \sum_{j=1}^{n} \varphi_{j} \leq a, \\
\delta \min _{s \in[\delta b, b]} f(s) \sum_{j=1}^{n} \varphi_{j} \geq b
\end{array}
$$

or

$$
\begin{gathered}
\delta \min _{s \in[\delta a, a]} f(s) \sum_{j=1}^{n} \varphi_{j} \geq a, \\
\max _{s \in[\delta b, b]} f(s) \sum_{j=1}^{n} \varphi_{j} \leq b
\end{gathered}
$$

hold.

Theorem 5. Assume that conditions $\left(A_{1}\right)$ and $\left(A_{2}\right)$ hold; the set $P=\left\{x \in R^{n}: x_{i} \geq \delta|x|\right.$ for $\left.i \in[1, n]\right\}$, and then problem (3) or (4) has at least a positive solution $x \in P \cap\left(\bar{\Omega}_{b} \backslash \Omega_{a}\right)$. Such solution satisfies the condition $a \leq|x| \leq b$.

Proof. For $\delta \in(0,1]$, clearly, the set

$$
P=\left\{x \in R^{n}: x_{i} \geq \delta|x| \text { for } i \in[1, n]\right\}
$$

is a cone of $R^{n}$, where $|x|=\max _{i \in[1, n]}\left|x_{i}\right|$.

For $0<a<b$, we denote that

$$
\begin{aligned}
& \Omega_{a}=\left\{x:|x|<a, x \in R^{n}\right\}, \\
& \Omega_{b}=\left\{x:|x|<b, x \in R^{n}\right\} .
\end{aligned}
$$

Then, for $x \in P \cap\left(\bar{\Omega}_{b} \backslash \Omega_{a}\right)$, we have

$$
y_{i}=\sum_{j=1}^{n} g_{i j} f\left(x_{j}\right) \leq \sum_{j=1}^{n} \varphi_{j} f\left(x_{j}\right) \quad \text { for } i \in[1, n]
$$

which implies that

$$
|y| \leq \sum_{j=1}^{n} \varphi_{j} f\left(x_{j}\right)
$$

On the other hand,

$$
y_{i}=\sum_{j=1}^{n} g_{i j} f\left(x_{j}\right) \geq \delta \sum_{j=1}^{n} \varphi_{j} f\left(x_{j}\right) \geq \delta|y|
$$

for $x \in P \cap\left(\bar{\Omega}_{b} \backslash \Omega_{a}\right)$. That is, $G F\left(P \cap\left(\bar{\Omega}_{b} \backslash \Omega_{a}\right)\right) \subset P$.

Note that the function $f(s)$ is continuous for $s \in[\delta a, b]$; clearly, $G F: P \cap\left(\bar{\Omega}_{b} \backslash \Omega_{a}\right) \rightarrow P$ is completely continuous.

For $x \in P \cap \partial \Omega_{a}$, we have

$$
y_{i}=\sum_{j=1}^{n} g_{i j} f\left(x_{j}\right) \leq \sum_{j=1}^{n} \varphi_{j} f\left(x_{j}\right) \leq \max _{s \in[\delta a, a]} f(s) \sum_{j=1}^{n} \varphi_{j}
$$

$\leq a$.
For $x \in P \cap \partial \Omega_{b}$, we have $\delta|x|=b \delta \leq x_{i} \leq b$ for $i \in[1, n]$ and

$$
y_{i}=\sum_{j=1}^{n} g_{i j} f\left(x_{j}\right) \geq \delta \sum_{j=1}^{n} \varphi_{j} f\left(x_{j}\right) \geq \delta \min _{s \in[\delta b, b]} f(s) \sum_{j=1}^{n} \varphi_{j}
$$

$\geq b$.

On the opposite, for $x \in P \cap \partial \Omega_{a}$ we have

$$
y_{i}=\sum_{j=1}^{n} g_{i j} f\left(x_{j}\right) \geq \delta \min _{s \in[\delta a, a]} f(s) \sum_{j=1}^{n} \varphi_{j} \geq a .
$$

For $x \in P \cap \partial \Omega_{b}$, we have

$$
y_{i}=\sum_{j=1}^{n} g_{i j} f\left(x_{j}\right) \leq \max _{s \in[\delta b, b]} f(s) \sum_{j=1}^{n} \varphi_{j} \leq b
$$

According to Lemma 1, we complete the proof.

Remark 6. Theorem 5 improves the corresponding result in [12] even if system (3) is reduced to problem (1). For example, when $n=5$, we have

$$
G=\frac{1}{6}\left(\begin{array}{lllll}
5 & 4 & 3 & 2 & 1 \\
4 & 8 & 6 & 4 & 2 \\
3 & 6 & 9 & 6 & 3 \\
2 & 4 & 6 & 8 & 4 \\
1 & 2 & 3 & 4 & 5
\end{array}\right),
$$

$$
\begin{aligned}
& \varphi=\frac{1}{6} \operatorname{col}(5,8,9,8,5), \\
& 0<\delta \leq \frac{1}{5}
\end{aligned}
$$

by using Theorem 5 . However, according to Theorem 1 in [12], $\delta^{\prime}=1 / 9$. Clearly, we have

$$
\begin{aligned}
& {\left[\frac{1}{5} a, a\right] \subset\left[\frac{1}{9} a, a\right],} \\
& {\left[\frac{1}{5} b, b\right] \subset\left[\frac{1}{9} b, b\right] .}
\end{aligned}
$$

Thus, we improve the recent result when system (3) is reduced to problem (1). 
Remark 7. When conditions (6) and (7) of $\left(\mathrm{A}_{2}\right)$ are, respectively, replaced by

$$
\begin{gathered}
\max _{s \in[\delta a, a]} f(s) \sum_{j=1}^{n} \varphi_{j}<a, \\
\delta \min _{s \in[\delta b, b]} f(s) \sum_{j=1}^{n} \varphi_{j} \geq b, \\
\delta \min _{s \in[\delta a, a]} f(s) \sum_{j=1}^{n} \varphi_{j}>a, \\
\max _{s \in[\delta b, b]} f(s) \sum_{j=1}^{n} \varphi_{j} \leq b, \\
\max _{s \in[\delta a, a]} f(s) \sum_{j=1}^{n} \varphi_{j} \leq a, \\
\delta \min _{s \in[\delta b, b]} f(s) \sum_{j=1}^{n} \varphi_{j}>b, \\
\delta \min _{s \in[\delta a, a]} f(s) \sum_{j=1}^{n} \varphi_{j} \geq a, \\
\max _{s \in[\delta b, b]} f(s) \sum_{j=1}^{n} \varphi_{j}<b,
\end{gathered}
$$

or

$$
\begin{array}{r}
\max _{s \in[\delta a, a]} f(s) \sum_{j=1}^{n} \varphi_{j}<a, \\
\delta \min _{s \in[\delta b, b]} f(s) \sum_{j=1}^{n} \varphi_{j}>b, \\
\delta \min _{s \in[\delta a, a]} f(s) \sum_{j=1}^{n} \varphi_{j}>a, \\
\max _{s \in[\delta b, b]} f(s) \sum_{j=1}^{n} \varphi_{j}<b,
\end{array}
$$

some corresponding results can be obtained by using Corollaries 2, 3, and 4 . The proof will be omitted.

When $n=1$, (3) is reduced to $x=f(x)$. In this case, we have the following result.

Corollary 8. Assume that there exist $0<a<b$, such that $f \in C[a, b]$ and either $f(a) \leq a$ and $f(b) \geq b$ or $f(a) \geq a$ and $f(b) \leq b$, then there is $x^{*} \in[a, b]$, such that $x^{*}=f\left(x^{*}\right)$; that is, the function $f(x)$ has a fixed point $x^{*} \in[a, b]$.

Remark 9. Clearly, Corollary 8 is sharp.
Example 10. Consider the nonlinear algebraic system of the form

$$
\left(\begin{array}{l}
x_{1} \\
x_{2}
\end{array}\right)=\left(\begin{array}{ll}
1 & 1 \\
1 & 1
\end{array}\right)\left(\begin{array}{l}
f\left(x_{1}\right) \\
f\left(x_{2}\right)
\end{array}\right)
$$

Then, we have $\varphi_{1}=\varphi_{2}=1, \delta=1$, and

$$
\begin{aligned}
P & =\left\{\left(x_{1}, x_{2}\right): x_{1}, x_{2} \geq \max \left\{\left|x_{1}\right|,\left|x_{2}\right|\right\},\left(x_{1}, x_{2}\right)\right. \\
& \left.\in R^{2}\right\} .
\end{aligned}
$$

In view of Theorem 5 , if there exists $0<a<b$ such that either $2 f(a) \leq a$ and $2 f(b) \geq b$ or $2 f(a) \geq a$ and $2 f(b) \leq b$ hold, then problem (21) has at least a positive solution $x \in$ $P \cap\left(\bar{\Omega}_{b} \backslash \Omega_{a}\right)$. Note that $\left(x_{1}, x_{2}\right) \in P$ implies that $x_{1}=x_{2}$; thus, we have $a \leq x_{1}=x_{2} \leq b$. In fact, problem (21) can be rewritten by

$$
\begin{aligned}
& x_{1}=f\left(x_{1}\right)+f\left(x_{2}\right), \\
& x_{2}=f\left(x_{1}\right)+f\left(x_{2}\right) .
\end{aligned}
$$

Let $x_{1}=x_{2}=x$; we have $x=2 f(x)$. Thus, in view of Corollary 8, problem (21) has at least a positive solution $x \in P \cap\left(\bar{\Omega}_{b} \backslash \Omega_{a}\right)$ with $a \leq x_{1}=x_{2} \leq b$. Such result is also sharp.

Remark 11. Note that the function $f$ may have no definition on $[0, \delta a)$. Thus, the function $f$ may have singularities. For example,

$$
f(x)=\frac{1}{x^{\alpha}},
$$

where $\alpha>0$. In $[8,13]$, it is assumed that $f$ is sublinear or superlinear at zero point and infinite point. In this situation, we can also obtain some corresponding results.

\section{More General Results}

In this section, we assume that the coefficient matrix $G$ and the nonlinear term of (3) may be dependent on the variable $x$. In this case, we have

$$
x=G(x) F(x)
$$

or

$$
x_{i}=\sum_{j=1}^{n} g_{i j}(x) f_{j}(x) \quad \text { for } i \in[1, n],
$$

where

$$
\begin{aligned}
& G(x)=\left(g_{i j}\left(x_{1}, x_{2}, \ldots, x_{n}\right)\right)_{n \times n}, \\
& F(x)=\operatorname{col}\left(f_{1}\left(x_{1}, \ldots, x_{n}\right), f_{2}\left(x_{1}, \ldots, x_{n}\right), \ldots,\right. \\
& \left.\quad f_{n}\left(x_{1}, \ldots, x_{n}\right)\right) .
\end{aligned}
$$


At this time, we give the following conditions:

$\left(\mathrm{S}_{1}\right)$ There exists a positive $b$, such that $g_{i j}(x)$ is continuous and nonnegative $0 \leq x_{i} \leq b$ and $i, j \in[1, n]$.

$\left(\mathrm{S}_{2}\right)$ There exist $\delta \in(0,1]$ and a positive sequence $\left\{\varphi_{j}\right\}_{j=1}^{n}$, such that $\delta \varphi_{j} \leq g_{i j}(x) \leq \varphi_{j}$ for $0 \leq x_{i} \leq b, i, j \in$ $[1, n]$.

$\left(\mathrm{S}_{3}\right)$ There exist $0<a<b$, such that the function $f_{i}$ : $[\delta a, b]^{n} \rightarrow R_{+}$is continuous and either

$$
\begin{gathered}
\max _{\substack{s_{t} \in[\delta a, a] \\
j \in[1, n]}} f_{j}\left(s_{1}, s_{2}, \ldots, s_{n}\right) \sum_{j=1}^{n} \varphi_{j} \leq a, \\
\delta \min _{\substack{s_{t} \in[\delta b, b] \\
j \in[1, n]}} f_{j}\left(s_{1}, s_{2}, \ldots, s_{n}\right) \sum_{j=1}^{n} \varphi_{j} \geq b
\end{gathered}
$$

or

$$
\begin{gathered}
\delta \min _{\substack{s_{t} \in[\delta a, a] \\
j \in[1, n]}} f_{j}\left(s_{1}, s_{2}, \ldots, s_{n}\right) \sum_{j=1}^{n} \varphi_{j} \geq a, \\
\max _{\substack{s_{t} \in[\delta b, b] \\
j \in[1, n]}} f_{j}\left(s_{1}, s_{2}, \ldots, s_{n}\right) \sum_{j=1}^{n} \varphi_{j} \leq b
\end{gathered}
$$

hold for $i \in[1, n]$.

Similarly, we have the following result.

Theorem 12. Assume that conditions $\left(S_{1}\right),\left(S_{2}\right)$, and $\left(S_{3}\right)$ hold; then problem (25) or (26) has at least a positive solution $x \in$ $P \cap\left(\bar{\Omega}_{b} \backslash \Omega_{a}\right)$. Such solution satisfies the condition $a \leq|x| \leq b$.

Proof. Similarly, we can prove that $G F=A: P \cap\left(\bar{\Omega}_{b} \backslash \Omega_{a}\right) \rightarrow$ $P$ is completely continuous.

For $x \in P \cap \partial \Omega_{a}$, we have

$$
\begin{aligned}
y_{i} & =\sum_{j=1}^{n} g_{i j}(x) f_{j}(x) \leq \sum_{j=1}^{n} \varphi_{j} f_{j}(x) \\
& \leq \max _{\substack{s_{t} \in[\delta a, a] \\
j \in[1, n]}} f_{j}\left(s_{1}, s_{2}, \ldots, s_{n}\right) \sum_{j=1}^{n} \varphi_{j} \leq a
\end{aligned}
$$

or

$$
\begin{aligned}
y_{i} & =\sum_{j=1}^{n} g_{i j}(x) f_{j}(x) \geq \delta \sum_{j=1}^{n} \varphi_{j} f_{j}(x) \\
& \geq \delta \min _{\substack{s_{t} \in[\delta a, a] \\
j \in[1, n]}} f_{j}\left(s_{1}, s_{2}, \ldots, s_{n}\right) \sum_{j=1}^{n} \varphi_{j} \geq a .
\end{aligned}
$$

For $x \in P \cap \partial \Omega_{b}$, the cases are similar. The proof is complete.
Example 13. Consider the nonlinear algebraic system of the form

$$
\left(\begin{array}{l}
p_{1} \\
p_{2}
\end{array}\right)=\left(\begin{array}{cc}
p_{1}+1 & 1 \\
1 & p_{2}+1
\end{array}\right)\left(\begin{array}{l}
f_{1}\left(p_{1}, p_{2}\right) \\
f_{2}\left(p_{1}, p_{2}\right)
\end{array}\right),
$$

where $p_{1}, p_{2} \in[0,1]$. We can choose that $b=1$ and $a=1 / 4$. At this time, we have $\varphi_{1}=\varphi_{2}=2$ and $\delta=1 / 2$ which imply that conditions $\left(S_{1}\right)$ and $\left(S_{2}\right)$ hold. At the same time, we have

$$
\begin{gathered}
\max _{s_{t} \in[\delta a, a]} f_{1}\left(s_{1}, s_{2}\right) \sum_{j=1}^{2} \varphi_{j}=4 \max _{s_{t} \in[1 / 8,1 / 4]} f_{1}\left(s_{1}, s_{2}\right), \\
\max _{s_{t} \in[\delta a, a]} f_{2}\left(s_{1}, s_{2}\right) \sum_{j=1}^{2} \varphi_{j}=4 \max _{s_{t} \in[1 / 8,1 / 4]} f_{2}\left(s_{1}, s_{2}\right), \\
\delta \min _{s_{t} \in[\delta b, b]} f_{1}\left(s_{1}, s_{2}\right) \sum_{j=1}^{2} \varphi_{j}=2 \min _{s_{t} \in[1 / 2,1]} f_{1}\left(s_{1}, s_{2}\right),
\end{gathered}
$$

$$
\delta \min _{s_{t} \in[\delta b, b]} f_{2}\left(s_{1}, s_{2}\right) \sum_{j=1}^{2} \varphi_{j}=2 \min _{s_{t} \in[1 / 2,1]} f_{2}\left(s_{1}, s_{2}\right) .
$$

In view of Theorem 12 , when

$$
\begin{gathered}
\max _{s_{t} \in[1 / 8,1 / 4]} f_{1}\left(s_{1}, s_{2}\right), \max _{s_{t} \in[1 / 8,1 / 4]} f_{2}\left(s_{1}, s_{2}\right) \leq \frac{1}{16}, \\
\min _{s_{t} \in[1 / 2,1]} f_{1}\left(s_{1}, s_{2}\right), \min _{s_{t} \in[1 / 2,1]} f_{2}\left(s_{1}, s_{2}\right) \geq \frac{1}{2},
\end{gathered}
$$

system (32) has at least a positive solution $\left(p_{1}^{*}, p_{2}^{*}\right) \in[1 / 8,1]^{2}$. For example, let

$$
\begin{aligned}
& f_{1}(x)= \begin{cases}x^{2}, & \frac{1}{8} \leq x \leq \frac{1}{4}, \\
\frac{15}{4} x-\frac{7}{8}, & \frac{1}{4} \leq x \leq 1,\end{cases} \\
& f_{2}(x)= \begin{cases}\frac{1}{4} x, & \frac{1}{8} \leq x \leq \frac{1}{4}, \\
-\frac{7}{3} x^{2}+\frac{11}{2} x-\frac{7}{6}, & \frac{1}{4} \leq x \leq 1 .\end{cases}
\end{aligned}
$$

We have

$$
\begin{aligned}
& \max _{s_{t} \in[1 / 8,1 / 4]} f_{1}\left(s_{1}, s_{2}\right) \leq \frac{1}{16}, \\
& \max _{s_{t} \in[1 / 8,1 / 4]} f_{2}\left(s_{1}, s_{2}\right) \leq \frac{1}{16}, \\
& \min _{s_{t} \in[1 / 2,1]} f_{1}\left(s_{1}, s_{2}\right) \geq \frac{1}{2}, \\
& \min _{s_{t} \in[1 / 2,1]} f_{2}\left(s_{1}, s_{2}\right) \geq \frac{1}{2} .
\end{aligned}
$$

Thus, all conditions of Theorem 12 hold. In [11, 23], it is assumed that $A$ is a positive or nonnegative matrix, where $a_{i j}$ is independent on the variable $x$. In the above section, the coefficient matrix $G$ is dependent on the variable $x$. 
Remark 14. Clearly, we can also obtain the existence conditions for the systems

$$
\left(\begin{array}{l}
p_{1} \\
p_{2}
\end{array}\right)=\left(\begin{array}{ll}
2 & 1 \\
1 & 2
\end{array}\right)\left(\begin{array}{l}
f_{1}\left(p_{1}, p_{2}\right) \\
f_{2}\left(p_{1}, p_{2}\right)
\end{array}\right)
$$

or

$$
\left(\begin{array}{l}
p_{1} \\
p_{2}
\end{array}\right)=\left(\begin{array}{cc}
p_{1}+1 & 1 \\
1 & p_{2}+1
\end{array}\right)\left(\begin{array}{l}
f_{1}\left(p_{1}\right) \\
f_{2}\left(p_{2}\right)
\end{array}\right) .
$$

They will be omitted.

Remark 15. When conditions (28) and (29) of $\left(\mathrm{S}_{3}\right)$ are, respectively, replaced by

$$
\begin{aligned}
& \max _{\substack{s_{t} \in[\delta a, a] \\
j \in[1, n]}} f_{j}\left(s_{1}, s_{2}, \ldots, s_{n}\right) \sum_{j=1}^{n} \varphi_{j}<a, \\
& \delta \min _{\substack{s_{t} \in[\delta b, b] \\
j \in[1, n]}} f_{j}\left(s_{1}, s_{2}, \ldots, s_{n}\right) \sum_{j=1}^{n} \varphi_{j} \geq b, \\
& \delta \min _{\substack{s_{t} \in[\delta a, a] \\
j \in[1, n]}} f_{j}\left(s_{1}, s_{2}, \ldots, s_{n}\right) \sum_{j=1}^{n} \varphi_{j}>a, \\
& \max _{\substack{s_{t} \in[\delta b, b] \\
j \in[1, n]}} f_{j}\left(s_{1}, s_{2}, \ldots, s_{n}\right) \sum_{j=1}^{n} \varphi_{j} \leq b, \\
& \max _{\substack{s_{t} \in[\delta a, a] \\
j \in[1, n]}} f_{j}\left(s_{1}, s_{2}, \ldots, s_{n}\right) \sum_{j=1}^{n} \varphi_{j} \leq a, \\
& \delta \min _{\substack{s_{t} \in[\delta b, b] \\
j \in[1, n]}} f_{j}\left(s_{1}, s_{2}, \ldots, s_{n}\right) \sum_{j=1}^{n} \varphi_{j}>b, \\
& \delta \min _{\substack{s_{t} \in[\delta a, a] \\
j \in[1, n]}} f_{j}\left(s_{1}, s_{2}, \ldots, s_{n}\right) \sum_{j=1}^{n} \varphi_{j} \geq a, \\
& \max _{\substack{s_{t} \in[\delta b, b] \\
j \in[1, n]}} f_{j}\left(s_{1}, s_{2}, \ldots, s_{n}\right) \sum_{j=1}^{n} \varphi_{j}<b,
\end{aligned}
$$

or

$$
\begin{gathered}
\max _{\substack{s_{t} \in[\delta a, a] \\
j \in[1, n]}} f_{j}\left(s_{1}, s_{2}, \ldots, s_{n}\right) \sum_{j=1}^{n} \varphi_{j}<a, \\
\delta \min _{\substack{s_{t} \in[\delta b, b] \\
j \in[1, n]}} f_{j}\left(s_{1}, s_{2}, \ldots, s_{n}\right) \sum_{j=1}^{n} \varphi_{j}>b, \\
\delta \min _{\substack{s_{t} \in[\delta a, a] \\
j \in[1, n]}} f_{j}\left(s_{1}, s_{2}, \ldots, s_{n}\right) \sum_{j=1}^{n} \varphi_{j}>a, \\
\max _{\substack{s_{t} \in[\delta b, b] \\
j \in[1, n]}} f_{j}\left(s_{1}, s_{2}, \ldots, s_{n}\right) \sum_{j=1}^{n} \varphi_{j}<b,
\end{gathered}
$$

some corresponding results can be obtained by using Corollaries 2, 3, and 4 .

Remark 16. Problem (25) can be rewritten by

$$
H(x)=G(x) F(x)-x=0 .
$$

In fact, we also obtain the existence of positive zero points for system (41). The existence of zero points for (41) has been extensively discussed; see [9, 20, 21, 24, 25].

\section{Conflict of Interests}

The authors declare that there is no conflict of interests regarding the publication of this paper.

\section{Acknowledgments}

This work was supported by the National Natural Science Foundation of China (no. 11371277) and the Natural Sciences and Engineering Research Council of Canada (NSERC).

\section{References}

[1] O. Carbonell-Nicolau, "On the existence of pure strategy perfect equilibrium in discontinuous games," Games and Economic Behavior, vol. 71, no. 1, pp. 23-48, 2011.

[2] C. A. Díaz, F. A. Campos, and J. Villar, "Existence and uniqueness of conjectured supply function equilibria," International Journal of Electrical Power \& Energy Systems, vol. 58, pp. 266273, 2014.

[3] W.-C. Guo, F.-C. Lai, and D.-Z. Zeng, "A Hotelling model with production," Mathematical Social Sciences, vol. 73, pp. 40-49, 2015.

[4] A. Tasnádi, "Existence of pure strategy nash equilibrium in bertrand-edgeworth oligopolies," Economics Letters, vol. 63, no. 2, pp. 201-206, 1999.

[5] X. Li, X. Wang, and G. Chen, "Pinning a complex dynamical network to its equilibrium," IEEE Transactions on Circuits and Systems I: Regular Papers, vol. 51, no. 10, pp. 2074-2087, 2004.

[6] Y. Shi and G. Chen, "Chaos of discrete dynamical systems in complete metric spaces," Chaos, Solitons and Fractals, vol. 22, no. 3, pp. 555-571, 2004.

[7] Y. Shi and G. Chen, "Chaos of discrete dynamical systems in Banach spaces," Science China Mathematics A, vol. 48, pp. 222238, 2005.

[8] P. J. Zufiria and R. S. Guttalu, "A computational method for finding all the roots of a vector function," Applied Mathematics and Computation, vol. 35, no. 1, pp. 13-59, 1990.

[9] M. I. Syam, "Nonlinear optimization exclusion tests for finding all solutions of nonlinear equations," Applied Mathematics and Computation, vol. 170, no. 2, pp. 1104-1116, 2005.

[10] W. Y. Feng and G. Zhang, "Eigenvalue and spectral intervals for a nonlinear algebraic system," Linear Algebra and its Applications, vol. 439, no. 1, pp. 1-20, 2013.

[11] G. Zhang and W. Y. Feng, "On the number of positive solutions of a nonlinear algebraic system," Linear Algebra and Its Applications, vol. 422, no. 2-3, pp. 404-421, 2007.

[12] G. Zhang and S. Ge, "Existence of positive solutions for a class of discrete Dirichlet boundary value problems," Applied Mathematics Letters, vol. 48, pp. 1-7, 2015. 
[13] G. Zhang and R. Medina, "Three-point boundary value problems for difference equations," Computers \& Mathematics with Applications, vol. 48, no. 12, pp. 1791-1799, 2004.

[14] G. van der Laan, D. Talman, and Z. Yang, "Solving discrete systems of nonlinear equations," European Journal of Operational Research, vol. 214, no. 3, pp. 493-500, 2011.

[15] A. Bagh, "Variational convergence: approximation and existence of equilibria in discontinuous games," Journal of Economic Theory, vol. 145, no. 3, pp. 1244-1268, 2010.

[16] G. Bigi, M. Castellani, M. Pappalardo, and M. Passacantando, "Existence and solution methods for equilibria," European Journal of Operational Research, vol. 227, no. 1, pp. 1-11, 2013.

[17] P. R. Chowdhury, "Bertrand-Edgeworth equilibrium large markets with non-manipulable residual demand," Economics Letters, vol. 79, no. 3, pp. 371-375, 2003.

[18] K. Deimling, Nonlinear Functional Analysis, Springer, New York, NY, USA, 1985.

[19] E. Einy, O. Haimanko, D. Moreno, and B. Shitovitz, "On the existence of Bayesian Cournot equilibrium," Games and Economic Behavior, vol. 68, no. 1, pp. 77-94, 2010.

[20] K. Mathia and R. Saeks, "Solving nonlinear equations using recurrent neural networks," in Proceedings of the World Congress on Neural Networks, Washington, DC, USA, July 1995.

[21] J. M. Soriano, "On the existence of zero points," Applied Mathematics and Computation, vol. 79, no. 1, pp. 99-104, 1996.

[22] M. Yannakakis, "Equilibria, fixed points, and complexity classes," Computer Science Review, vol. 3, no. 2, pp. 71-85, 2009.

[23] H. Wang, M. Wang, and E. Wang, "An application of the Krasnoselskii theorem to systems of algebraic equations," Journal of Applied Mathematics and Computing, vol. 38, no. 1-2, pp. 585600, 2012.

[24] A. Galántai and A. Jeney, "Quasi-Newton ABS methods for solving nonlinear algebraic systems of equations," Journal of Optimization Theory and Applications, vol. 89, no. 3, pp. 561$573,1996$.

[25] K. Goulianas, A. Margaris, and M. Adamopoulos, "Finding all real roots of $3 \times 3$ nonlinear algebraic systems using neural networks," Applied Mathematics and Computation, vol. 219, no. 9, pp. 4444-4464, 2013.

[26] T. Gao, H. Wang, and M. Wu, "Nonnegative solutions of a class of systems of algebraic equations," Dynamic Systems and Applications, vol. 23, no. 2-3, pp. 211-219, 2014.

[27] N. M. Madbouly, D. F. McGhee, and G. F. Roach, "Adomian's method for Hammerstein integral equations arising from chemical reactor theory," Applied Mathematics and Computation, vol. 117, no. 2-3, pp. 241-249, 2001.

[28] J. Schauder, "Der fixpunktsatz in funktionalraümen," Studia Mathematica, vol. 2, no. 1, pp. 171-180, 1930.

[29] A. Saadatmandi, M. Razzaghi, and M. Dehghan, "Sinc-Galerkin solution for nonlinear two-point boundary value problems with applications to chemical reactor theory," Mathematical and Computer Modelling, vol. 42, no. 11-12, pp. 1237-1244, 2005.

[30] D. J. Guo and V. Lakshmikantham, Nonlinear Problems in Abstract Cones, Academic Press, San Diego, Calif, USA, 1988. 


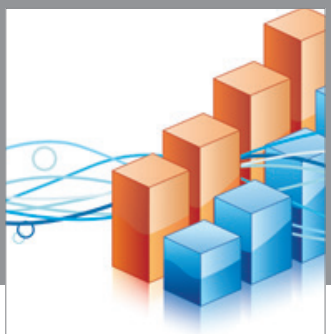

Advances in

Operations Research

vatem alat4

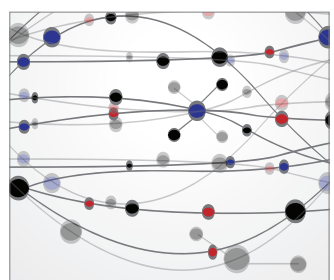

\section{The Scientific} World Journal
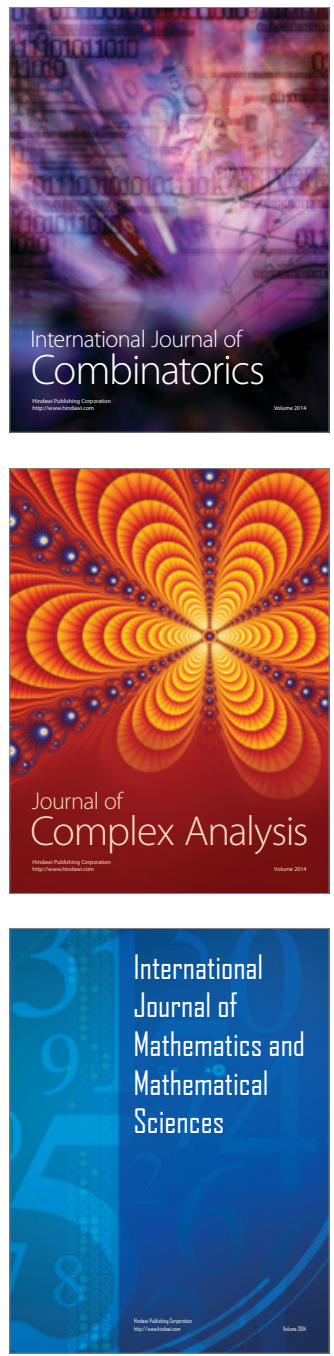
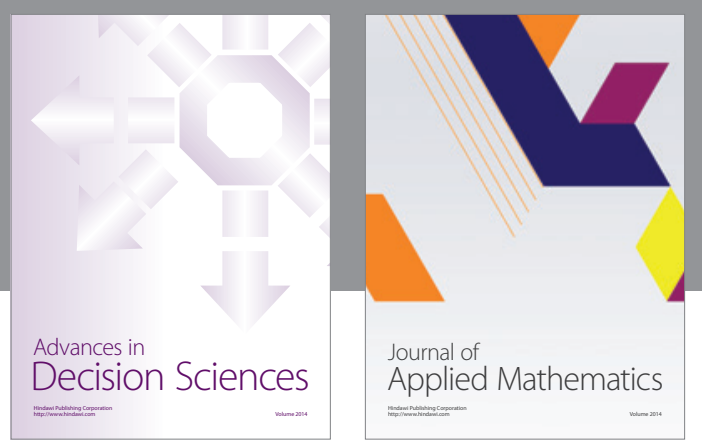

Algebra

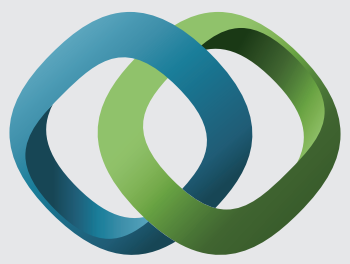

\section{Hindawi}

Submit your manuscripts at

http://www.hindawi.com
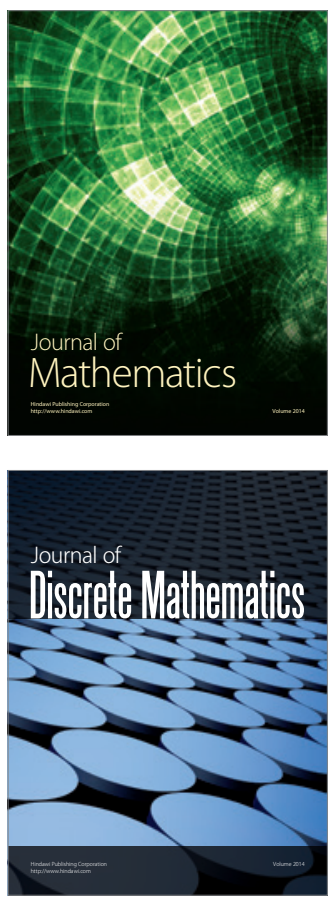

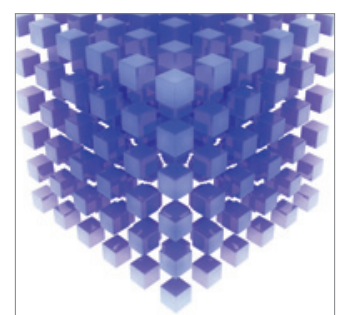

Mathematical Problems in Engineering
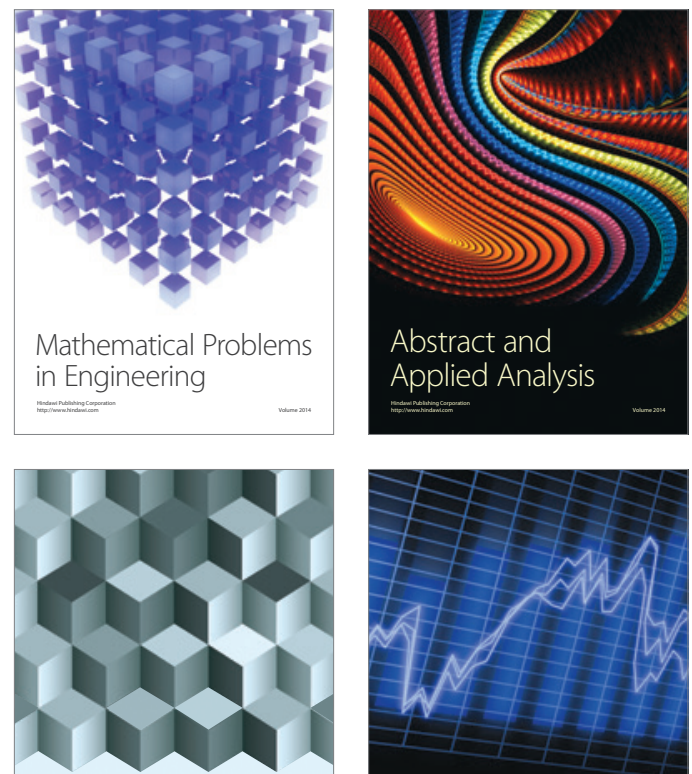

Journal of

Function Spaces

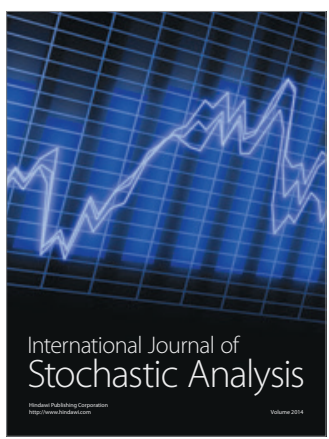

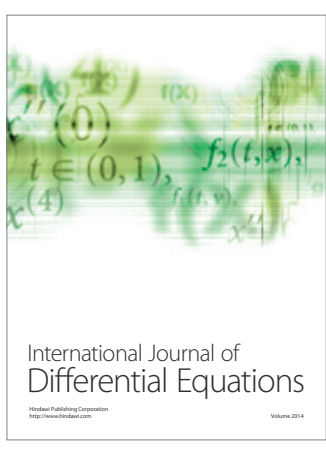
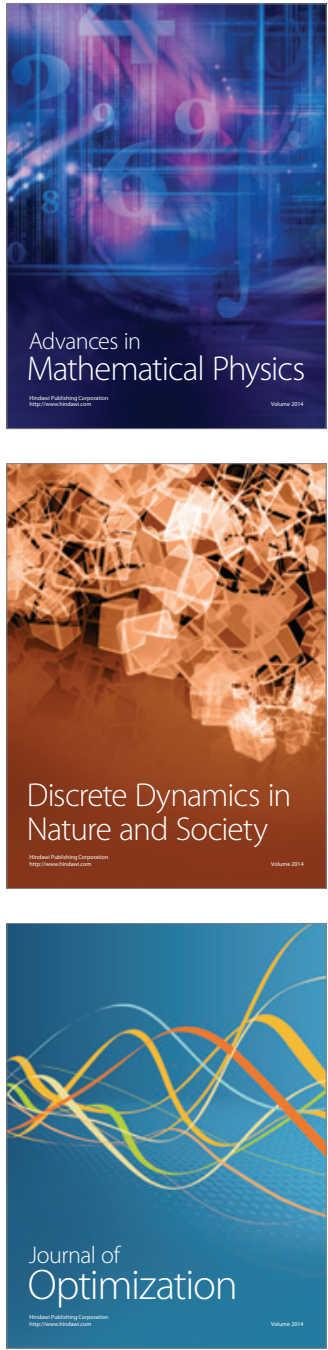